\title{
The relationship between body composition and knee osteoarthritis in postmenopausal women
}

\author{
Hakan Nur $(\mathbb{0}$, Tiraje Tuncer $(1)$ \\ Department of Physical Medicine and Rehabilitation, Medicine Faculty of Akdeniz University, Antalya, Turkey
}

Received: March 17, 2017 Accepted: July 18, 2017 Published online: March 03, 2018

\begin{abstract}
Objectives: The aim of this study was to examine the relationship between the body composition measures and knee osteoarthritis (OA) in postmenopausal women to determine whether the fat mass or the lean mass was closely associated with knee OA.

Patients and methods: This retrospective, cross-sectional study included a total of 212 postmenopausal women (mean age $59.9 \pm 6.2$ years; range, 46 to 76 years). Descriptive characteristics were recorded and height was measured using a stadiometer. Body weight, fat mass, and lean mass were estimated using bioelectrical impedance analysis. X-rays of the knees were obtained. Knee OA was radiographically diagnosed according to the Kellgren-Lawrence criteria. Based on the radiographic knee OA diagnosis the women were divided into two groups. Logistic regression analyses were used to examine the associations between the body composition measures and knee OA.

Results: Knee OA was present in 117 women. Body Mass Index, body weight, fat mass, and lean mass were all significantly associated with knee OA after adjusting for confounding factors such as age, height, and physical activity $(\mathrm{p}<0.001)$. When assessed together with lean mass in a multivariate regression model, fat mass was closely and significantly found to be related to knee OA, compared to lean mass ( $\mathrm{p}<0.001$, OR: 1.17). Furthermore the fat mass/lean mass ratio was positively associated with knee OA $(\mathrm{p}<0.001)$.

Conclusion: In postmenopausal women body weight was significantly and positively associated with knee OA and among the components that constitute body weight, fat mass was closely and significantly associated with knee OA compared to lean mass. This result suggests that the relationship between obesity and knee OA is mainly based on the fat mass, and supports the importance of systemic metabolic effects of adiposity in this relationship.
\end{abstract}

Keywords: Body composition; fat mass; knee osteoarthritis; lean mass; postmenopausal women.

Knee osteoarthritis (OA) is a common and disabling condition in the middle-aged adults and elderly and its prevalence has been growing with the increasing age of the population. ${ }^{[1,2]}$ Obesity is a well-known risk factor for knee OA. Increased Body Mass Index (BMI) is associated with an increased risk of radiographic and clinical knee OA, particularly in women. ${ }^{[3]}$ Although there is an evident relationship between obesity and knee $\mathrm{OA}$, the exact mechanism of this relationship is still unclear.

Biomechanical impact of increased body weight is considered to have an important role in this relationship. ${ }^{[4]}$ Being overweight is associated with the excess load and forces across a weight-bearing joint that can cause a damaged joint tissue. ${ }^{[5]}$ However, obesity is also associated with $\mathrm{OA}$ of non-weight -bearing joints such as joints of hand ${ }^{[6]}$ which suggests that the relationship between $\mathrm{OA}$ and obesity may not be explained only by biomechanical stress. Body weight is composed of three components mainly; fat mass, lean mass, and bone mass. Recent studies have shown that fat mass and fat percentage are significantly associated with knee $\mathrm{OA}$ and knee cartilage defects in women. ${ }^{[7,8]}$ However some other studies have demonstrated no such association. ${ }^{[9,10]}$ In addition, skeletal muscle mass, a surrogate of lean mass, has been reported to be positively associated with knee OA. ${ }^{[1]}$

Body composition changes in women with aging and menopausal status even in the absence of a alteration in body weight. Fat mass increases and 
lean mass decreases with aging, and there is a decline of lean mass with menopause. ${ }^{[12,13]}$ The incidence and severity of OA is higher in women, and more severe knee OA are seen after the menopause. ${ }^{[14,15]}$ A better understanding of the relationship between the body composition and knee OA, particularly in postmenopausal women, would be beneficial to elucidate the interaction between the obesity and knee OA. However, there is a lack of data evaluating the relationship between the body composition measures and knee OA in postmenopausal women.

In this study, we, therefore, aimed to investigate the relationship between the body composition and knee $\mathrm{OA}$ in a sample of Turkish postmenopausal women to determine whether the fat mass or the lean mass was closely associated with knee OA.

\section{PATIENTS AND METHODS}

This retrospective, cross-sectional study included data of 212 postmenopausal women (mean age 59.9 \pm 6.2 years; range, 46 to 76 years) who were admitted to our outpatient clinics between October 2014 and January 2016. Women whounderwent radiographic examination of the knees, body composition measurements by bioelectric impedance analysis, experienced natural menopause for at least one year and, completed questionnaires on demographic and clinical data were eligible for the study. Those with comorbidities which could affect the body composition, such as concurrent inflammatory rheumatic diseases, cardiovascular, pulmonary, renal, endocrine, neurologic disorders, malignancies, also those who had previously been or were presently taking drugs such as estrogen, glucocorticoids, thyroid hormones, those who were on diet or losing or gaining weight, and those who were having muscle-strengthening exercises were excluded. The study protocol was approved by the local Ethics Committee. The study was conducted in accordance with the principles of the Declaration of Helsinki.

Demographic and clinical data including physical activity levels were recorded. Women participating in physical activity such as walking, running, and cycling for more than $20 \mathrm{~min}$ and three days a week were considered physically active. Anthropometric measurements were obtained. Body composition measurements were performed through bioelectric impedance analysis (Body Composition Analyzer MC-780, Tanita Corp., Tokyo, Japan), according to the manufacturer's instructions. Body weight $(\mathrm{kg})$, fat mass $(\mathrm{kg})$, and lean mass $(\mathrm{kg})$ were measured. Height was measured to the nearest $0.1 \mathrm{~cm}$. Body Mass Index was calculated as the body weight divided by the square of the height $\left(\mathrm{kg} / \mathrm{m}^{2}\right)$.

Radiographic assessment was done by analyzing the bilateral weight-bearing anteroposterior and lateral plain radiographs of the participants. The radiographic changes related to OA were evaluated by two clinicians according to the Kellgren-Lawrence grading system. ${ }^{[16]}$ Participants were defined as having radiographic knee $\mathrm{OA}$, when they had grade $\geq 2$ radiography. Participants who had knee joint replacement and insufficient quality images were excluded. The women were divided into two groups according to the radiographic knee OA diagnosis; women with and without knee OA.

\section{Statistical analyses}

Statistical analysis was performed using the IBM SPSS version 23.0 software (IBM Corp., Armonk, NY, USA). Descriptive statistics were presented in mean \pm standard deviation (SD), and frequency, as appropriate. Univariate and multivariate logistic regression analyses were used to assess the relationship between body composition measures and knee OA. The presence/absence of knee OA was used as the outcome measure. Body weight, BMI, fat mass, lean mass, and fat/lean mass ratio were independent variables. In the univariate analyses, the relationship between knee OA and independent variables were assessed. In the multivariate analyses, independent variables were adjusted for the potential confounders of age, height, and physical activity, where appropriate. A further multivariate analysis was also performed including both fat mass and lean mass to evaluate the independent association of these variables with knee OA. Enter method was used and maximum five independent variables were entered to the models which provides requirement to perform logistic regression analyses. The overall

Table 1. Characteristics of the study population

\begin{tabular}{|c|c|c|c|}
\hline & $\mathrm{n}$ & $\%$ & Mean \pm SD \\
\hline Age (year) & & & $59.9 \pm 6.2$ \\
\hline Weight (kg) & & & $70.5 \pm 11.7$ \\
\hline Height (cm) & & & $155.0 \pm 5.1$ \\
\hline BMI $\left(\mathrm{kg} / \mathrm{m}^{2}\right)$ & & & $29.4 \pm 4.7$ \\
\hline Fat mass $(\mathrm{kg})$ & & & $26.4 \pm 7.5$ \\
\hline Lean mass (kg) & & & $41.9 \pm 4.9$ \\
\hline Fat mass / lean mass & & & $0.6 \pm 0.1$ \\
\hline \multicolumn{4}{|l|}{ Knee osteoarthritis } \\
\hline None & 95 & 45 & \\
\hline Osteoarthritis & 117 & 55 & \\
\hline \multicolumn{4}{|l|}{ Physical activity level } \\
\hline Non-active & 131 & 61.8 & \\
\hline Active & 81 & 38.2 & \\
\hline
\end{tabular}


Table 2. Relationship between the body composition measures and knee osteoarthritis

\begin{tabular}{|c|c|c|c|c|c|c|}
\hline & Univariate analysis & & Multivariate analysis* & & Multivariate analysis ${ }^{* *}$ & \\
\hline & OR (95\% CI) & $p$ & OR (95\% CI) & $p$ & OR (95\% CI) & $p$ \\
\hline Weight (kg) & 1.13 (1.09 to 1.17$)$ & $<0.001$ & 1.14 (1.10 to 1.18$)$ & $<0.001$ & & \\
\hline $\operatorname{BMI}\left(\mathrm{kg} / \mathrm{m}^{2}\right)$ & $1.36(1.24$ to 1.48$)$ & $<0.001$ & $1.34(1.22$ to 1.47$)$ & $<0.001$ & & \\
\hline Fat mass $(\mathrm{kg})$ & 1.21 (1.15 to 1.29$)$ & $<0.001$ & $1.22(1.15$ to 1.30$)$ & $<0.001$ & $1.17(1.08$ to 1.26$)$ & $<0.001$ \\
\hline Lean mass (kg) & $1.23(1.14$ to 1.33$)$ & $<0.001$ & $1.31(1.20$ to 1.44$)$ & $<0.001$ & $1.12(0.99$ to 1.25$)$ & 0.07 \\
\hline Fat mass / lean mass & $1.10(1.06$ to 1.13$)$ & $<0.001$ & 1.09 (1.06 to 1.12$)$ & $<0.001$ & & \\
\hline
\end{tabular}

* Odds ratio of knee osteoarthritis per unit increase in the body composition measures after adjustment for age, height, and physical activity levels (age and physical activity levels for $\mathrm{BMI}) ;{ }^{* *}$ Odds ratio of knee osteoarthritis per unit increase in fat mass and lean mass after adjustment for lean mass and fat mass including age, height, and physical activity levels; OR: Odds ratio; CI: Confidence interval; BMI: Body Mass Index;

classification of the models was between 67.9 and $72.2 \%$ for the univariate analyses, and between 72.6 and $75.5 \%$ for the multivariate analyses. The strength of the relationship between independent variables and knee OA was expressed in odds ratios (OR) with $95 \%$ confidence interval (CI) per unit increase in each variable. A $p$ value of $<0.05$ was considered statistically significant.

\section{RESULTS}

Radiographic knee OA was present in 117 women. Table 1 presents the characteristics of the study population including age, weight, height, BMI, fat mass, lean mass, fat mass/lean mass ratio, and physical activity levels.

The logistic regression models for assessing the associations between body composition measures and knee OA are presented in Table 2. In the univariate analysis, body weight, BMI, fat mass, lean mass, and fat mass/lean mass ratio were all significantly and positively associated with knee OA. In the multivariate analysis which was adjusted for age, height, and physical activity level, all variables remained significantly associated. After adjustment for fat mass and lean mass together, fat mass remained significantly and positively associated with knee OA $(\mathrm{p}<0.001)$, while lean mass was not found to be significantly associated with knee OA ( $\mathrm{p}>0.05)$.

\section{DISCUSSION}

In the present study, we investigated the relationship between body composition and knee OA in postmenopausal women. Body weight was positively associated with knee OA. Fat mass and lean mass, two components of body weight, were also significantly associated with knee OA; however, when adjusted together, fat mass became significantly and more closely associated with knee OA, compared to lean mass. In addition, a high fat mass/lean mass ratio was found unfavorable for knee OA.

Our results support the importance of adipose tissue in the relationship between body composition and knee $\mathrm{OA}$. The impact of obesity and excessive weight on knee OA are considered to occur due to different mechanisms such as; weight-bearing effects with excessive load on the knee joints, weight independent systemic metabolic effects or a combination of both. As fat mass remained positively and was significantly associated with knee OA after adjustment with lean mass and the significant association of lean mass with knee OA disappeared after adjustment with fat mass, we could argue that the relationship between obesity and knee $\mathrm{OA}$ is based on the fat mass in postmenopausal women and the systemic metabolic effects of adipose tissue seems to be more important in this relationship. If weight-bearing effects were the main factor, we would expect a similar impact of lean mass.

The findings of the study are in consistent with the recent studies evaluating the relationship between body composition and knee OA. Berry et al. ${ }^{[17]}$ reported a positive association between fat mass and bone marrow edema and suggested that inflammatory factors released from the adipose tissue might contribute to the vascular changes seen in these lesions. In healthy adults, a negative effect of fat mass was found on knee cartilage properties and fat mass was considered to be an important factor in the development and progression of cartilage defects. ${ }^{[18]}$ Visser et al. ${ }^{[7]}$ reported fat mass and low skeletal muscle percentage to be significantly associated with knee $\mathrm{OA}$ in women, suggesting that metabolic syndrome and systemic inflammation seen in individuals with greater adiposity may cause it.

There is also growing evidence concerning the metabolic link between adiposity and OA which may clarify the possible causes of our result. Adipose tissue is defined as an active metabolic and endocrine 
organ. It releases adipokines and also cytokines like interleukin-6 (IL-6) and tumor necrosis factor- $\alpha$ (TNF- $\alpha$ ) which potentially causes lowlevel systemic inflammation in excess body fat. ${ }^{[19,20]}$ A number of adipokines have been detected to mediate inflammatory effects and have catabolic roles in the pathophysiology of OA. ${ }^{[21,22]}$ Serum leptin levels were associated with MRI assessed cartilage defects and knee joint damage in middle-aged women. ${ }^{[23]}$ Leptin has been shown to increase the production of catabolic enzymes and proinflammatory mediators, such as matrix metalloproteinases, nitric oxide, IL-6, IL-8, and prostaglandin E2 in the cartilage. ${ }^{[24,25]}$ Visfatin, another adipokin, was found significantly greater in knee osteoarthritic patients, compared to the control individuals, particularly in advanced knee OA grades and was found to be positively correlated with degradation biomarkers of $\mathrm{OA} \cdot{ }^{[26]}$ Increased serum adiponectin were seen in female patients with erosive OA. ${ }^{[27]}$

Our study also showed that a high fat mass/lean mass ratio was unfavorable for knee OA in postmenopausal women. Weight loss is highly recommended for the non-surgical management of knee OA. ${ }^{[28]}$ It may be more beneficial, if it originates from fat mass in postmenopausal women. Rehabilitative approaches that provide losing fat mass and maintaining or improving muscle mass in overweight and obese postmenopausal women seems to be protective for the risk of knee OA. In a study evaluating weight loss, exercise and physical function in obese older adults, a combination of weight loss and exercise which provided more decrease in fat mass, compared to lean mass, resulted in greater improvement in physical function measures. ${ }^{[29]}$ Fat mass change was found to be related to symptomatic relief of obese patients with knee OA. ${ }^{[30]}$ A greater fat loss was also associated with greater gain in muscle strength and quality in older obese adults with knee $\mathrm{OA}$ in a weight loss intervention combined with exercise. ${ }^{[31]}$

Another finding is that, rather than the body weight or BMI, body composition is important in the relationship between obesity and knee OA in women. Obesity is defined by BMI which is calculated by using body weight and height. However, BMI does not differentiate adipose and non-adipose body mass and consider obesity-related characteristics. Individuals with lower body weight would be at a higher risk of OA with an unfavorable body composition. ${ }^{[32]}$

Our study has certain limitations. Cross-sectional design of the study may be insufficient to evaluate the causal relationship between body composition and knee OA. The body composition differences could have been influenced from knee OA. Longitudinal studies evaluating the relationship between the alteration of body composition and knee OA would be better. In addition, using bioelectric impedance analysis can be considered as a limitation, although it has good correlations with dual-energy X-ray absorptiometry for the assessment of body composition measures. ${ }^{[3]]}$ As the cohort of this study is the Turkish postmenopausal women, the ethnic differences seen in adiposity and body composition ${ }^{[34]}$ would have affect the relationship with knee OA. On the other hand, in evaluating the relationship between the body composition measures and knee OA, we used potential confounders for adjustment such as physical activity, which is known to affect body composition by losing fat mass and maintaining muscle mass which is a surrogate of lean mass. ${ }^{[35]}$

In conclusion this study shows that body weight is significantly and positively associated with knee $\mathrm{OA}$ and, among the components that constitute body weight, fat mass is closely and significantly associated with knee OA in postmenopausal women when assessed together with lean mass. This result suggests that the relationship between obesity and knee OA is mainly based on the fat mass, and supports the importance of systemic metabolic effects of adiposity in this relationship. In addition, a high fat mass/lean mass ratio is unfavorable for knee OA. In clinical practice, losing fat mass and maintaining or improving lean mass must be aimed in overweight and obese postmenopausal women to prevent from knee OA. Further studies should aim to elucidate the roles of inflammatory cytokines and adipokines in the development of knee OA and also evaluate the effects of rehabilitative interventions which aim reducing the fat mass and maintaining or improving the lean mass in overweight and obese postmenopausal women to prevent knee OA.

\section{Declaration of conflicting interests}

The authors declared no conflicts of interest with respect to the authorship and/or publication of this article.

\section{Funding}

The authors received no financial support for the research and/or authorship of this article.

\section{REFERENCES}

1. Guccione AA, Felson DT, Anderson JJ, Anthony JM, Zhang Y, Wilson PW, et al. The effects of specific medical conditions on the functional limitations of elders in the Framingham Study. Am J Public Health 1994;84:351-8. 
2. Lawrence RC, Felson DT, Helmick CG, Arnold LM, Choi $\mathrm{H}$, Deyo RA, et al. Estimates of the prevalence of arthritis and other rheumatic conditions in the United States. Part II. Arthritis Rheum 2008;58:26-35.

3. Jiang L, Tian W, Wang Y, Rong J, Bao C, Liu Y, et al. Body mass index and susceptibility to knee osteoarthritis: a systematic review and meta-analysis. Joint Bone Spine 2012;79:291-7.

4. Bennell KL, Bowles KA, Wang Y, Cicuttini F, Davies-Tuck M, Hinman RS. Higher dynamic medial knee load predicts greater cartilage loss over 12 months in medial knee osteoarthritis. Ann Rheum Dis 2011;70:1770-4.

5. Brandt KD, Dieppe P, Radin E. Etiopathogenesis of osteoarthritis. Med Clin North Am 2009;93:1-24.

6. Oliveria SA, Felson DT, Cirillo PA, Reed JI, Walker AM. Body weight, body mass index, and incident symptomatic osteoarthritis of the hand, hip, and knee. Epidemiology 1999;10:161-6.

7. Visser AW, de Mutsert R, Loef M, le Cessie S, den Heijer M, Bloem JL, et al. The role of fat mass and skeletal muscle mass in knee osteoarthritis is different for men and women: the NEO study. Osteoarthritis Cartilage 2014;22:197-202.

8. Ho-Pham LT, Lai TQ, Mai LD, Doan MC, Nguyen TV. Body Composition in Individuals with Asymptomatic Osteoarthritis of the Knee. Calcif Tissue Int 2016;98:165-71.

9. Abbate LM, Stevens J, Schwartz TA, Renner JB, Helmick CG, Jordan JM. Anthropometric measures, body composition, body fat distribution, and knee osteoarthritis in women. Obesity (Silver Spring) 2006;14:1274-81.

10. Hochberg MC, Lethbridge-Cejku M, Scott WW Jr, Reichle R, Plato CC, Tobin JD. The association of body weight, body fatness and body fat distribution with osteoarthritis of the knee: data from the Baltimore Longitudinal Study of Aging. J Rheumatol 1995;22:488-93.

11. Sowers MF, Yosef M, Jamadar D, Jacobson J, Karvonen-Gutierrez C, Jaffe M. BMI vs. body composition and radiographically defined osteoarthritis of the knee in women: a 4-year follow-up study. Osteoarthritis Cartilage 2008;16:367-72.

12. St-Onge MP. Relationship between body composition changes and changes in physical function and metabolic risk factors in aging. Curr Opin Clin Nutr Metab Care 2005;8:523-8.

13. Douchi T, Oki T, Nakamura S, Ijuin H, Yamamoto S, Nagata Y. The effect of body composition on bone density in pre- and postmenopausal women. Maturitas 1997;27:55-60.

14. O'Connor MI. Osteoarthritis of the hip and knee: sex and gender differences. Orthop Clin North Am 2006;37:55968.

15. Srikanth VK, Fryer JL, Zhai G, Winzenberg TM, Hosmer D, Jones G. A meta-analysis of sex differences prevalence, incidence and severity of osteoarthritis. Osteoarthritis Cartilage 2005;13:769-81.

16. Kellgren JH, Lawrence JS. Radiological assessment of osteoarthrosis. Ann Rheum Dis 1957;16:494-502.

17. Berry PA, Wluka AE, Davies-Tuck ML, Wang Y, Strauss BJ, Dixon JB, et al. The relationship between body composition and structural changes at the knee. Rheumatology (Oxford) 2010;49:2362-9.

18. Wang Y, Wluka AE, English DR, Teichtahl AJ, Giles GG, O'Sullivan R, et al. Body composition and knee cartilage properties in healthy, community-based adults. Ann Rheum Dis 2007;66:1244-8.

19. Kershaw EE, Flier JS. Adipose tissue as an endocrine organ. J Clin Endocrinol Metab 2004;89:2548-56.
20. Hotamisligil GS. Inflammation and metabolic disorders. Nature 2006;444:860-7.

21. Hu PF, Bao JP, Wu LD. The emerging role of adipokines in osteoarthritis: a narrative review. Mol Biol Rep 2011;38:873-8.

22. Daghestani HN, Kraus VB. Inflammatory biomarkers in osteoarthritis. Osteoarthritis Cartilage 2015;23:1890-6.

23. Karvonen-Gutierrez CA, Harlow SD, Jacobson J, Mancuso $\mathrm{P}$, Jiang Y. The relationship between longitudinal serum leptin measures and measures of magnetic resonance imagingassessed knee joint damage in a population of mid-life women. Ann Rheum Dis 2014;73:883-9.

24. Koskinen A, Vuolteenaho $\mathrm{K}$, Nieminen $\mathrm{R}$, Moilanen $\mathrm{T}$, Moilanen E. Leptin enhances MMP-1, MMP-3 and MMP-13 production in human osteoarthritic cartilage and correlates with MMP-1 and MMP-3 in synovial fluid from OA patients. Clin Exp Rheumatol 2011;29:57-64.

25. Vuolteenaho K, Koskinen A, Kukkonen M, Nieminen R, Päivärinta U, Moilanen T, et al. Leptin enhances synthesis of proinflammatory mediators in human osteoarthritic cartilage-mediator role of NO in leptin-induced PGE2, IL-6, and IL-8 production. Mediators Inflamm 2009;2009:345838.

26. Duan Y, Hao D, Li M, Wu Z, Li D, Yang X, et al. Increased synovial fluid visfatin is positively linked to cartilage degradation biomarkers in osteoarthritis. Rheumatol Int 2012;32:985-90.

27. Filková $M$, Lisková $M$, Hulejová $H$, Haluzík M, Gatterová J, Pavelková A, et al. Increased serum adiponectin levels in female patients with erosive compared with non-erosive osteoarthritis. Ann Rheum Dis 2009;68:295-6.

28. McAlindon TE, Bannuru RR, Sullivan MC, Arden NK, Berenbaum F, Bierma-Zeinstra SM, et al. OARSI guidelines for the non-surgical management of knee osteoarthritis. Osteoarthritis Cartilage 2014;22:363-88.

29. Villareal DT, Chode S, Parimi N, Sinacore DR, Hilton T, Armamento-Villareal R, et al. Weight loss, exercise, or both and physical function in obese older adults. N Engl J Med 2011;364:1218-29.

30. Toda Y, Toda T, Takemura S, Wada T, Morimoto T, Ogawa R. Change in body fat, but not body weight or metabolic correlates of obesity, is related to symptomatic relief of obese patients with knee osteoarthritis after a weight control program. J Rheumatol 1998;25:2181-6.

31. Wang X, Miller GD, Messier SP, Nicklas BJ. Knee strength maintained despite loss of lean body mass during weight loss in older obese adults with knee osteoarthritis. J Gerontol A Biol Sci Med Sci 2007;62:866-71.

32. Lee S, Kim TN, Kim SH. Sarcopenic obesity is more closely associated with knee osteoarthritis than is nonsarcopenic obesity: a cross-sectional study. Arthritis Rheum 2012;64:3947-54.

33. Thomson R, Brinkworth GD, Buckley JD, Noakes M, Clifton PM. Good agreement between bioelectrical impedance and dual-energy X-ray absorptiometry for estimating changes in body composition during weight loss in overweight young women. Clin Nutr 2007;26:771-7.

34. Leslie WD, Weiler HA, Nyomba BL. Ethnic differences in adiposity and body composition: the First Nations bone health study. Appl Physiol Nutr Metab 2007;32:1065-72.

35. Carnero EA, Dubis GS, Hames KC, Jakicic JM, Houmard JA, Coen $\mathrm{PM}$, et al. Randomized trial reveals that physical activity and energy expenditure are associated with weight and body composition after RYGB. Obesity (Silver Spring) 2017;25:1206-16. 